\title{
5.9 Left Ventricular Asymmetry in Hypertension: Effects on the
}

\section{QT Dispersion}

G. Ratti (1), F. Buono (1), C. Fulgione (2), C. Tedeschi (2), G. Covino (1),

P. Tammaro (1), G. Ricciardi (2), M. Volpicelli (1), F. Borrelli (2), P. Capogrosso (1)

(1)Cardiologia/UTIC - POS. G. Bosco - ASL NA 1, Napoli; (2)Cardiologia - POS

Gennaro - ASL NA 1, Napoli, Italy

Introduction. Several studies have shown that the left ventricular geometry can influence QT interval. Particularly, the left ventricular asymmetry it is in partnership to a greater dispersion of the interval QT in the subjects with left ventricular hypertrophy.

Aim. To verify the existing relationships among left ventricular asymmetry, left ventricular mass and QT dispersion in hypertensive patients with left ventricular hypertrophy (LVH).

Methods. 72 Caucasian patients have been studied, with I-II stage ESH-ESC hypertension (44 males and 28 females, middle aged $54 \pm 17$ years) and with body mass index (BMI) $28 \pm 5 \mathrm{~kg} / \mathrm{m} 2$. Were excluded: verified cardiovascular diseases, endocrine system diseases (included diabetes), and familiarity for sudden death. Homogeneous for life-style, and in absence of therapy, were submitted to clinical examination, electrocardiogram with evaluation of QT corrected dispersion (QTcD) and echocardiographic exam with evaluation of the index of asymmetry (AI) (analyzing the wall thickness in 13 segments with following relationship between the maximum and minimum wall thickness observed) assuming a value of 1,3 as limit among symmetrical and asymmetrical hypertrophy; values of left ventricular mass index (LVMI) exceeding $125 \mathrm{~g} / \mathrm{m} 2$ were considered for the diagnosis of hypertrophy. All patients have been classified in 2 groups: group A with asymmetrical LVH (30 patients) and group B without asymmetry (36 patients). Values of QTcD, age, LVMI and BMI have been compared between these two groups. Results have been expressed as middle mean \pm SD; statistical analysis was performed with Student's t-test for unpaired data, and a p-value $<0.05$ was considered statistically significant.

Results. Statistically meaningful differences were observed in values of QTcD (group A $=60 \pm 21 \mathrm{~ms}$ versus group $B=47 \pm 22 \mathrm{~ms}, \mathrm{p}<0.05$ ) and LVMI (group $\mathrm{A}=168 \pm 33 \mathrm{~g} / \mathrm{m} 2$ versus group $\mathrm{B}=151 \pm$ $24 \mathrm{~g} / \mathrm{m} 2 \mathrm{p}<0.05$ ), but not for age (group $A=52 \pm 18$ years versus group $B=55 \pm 15$ years, $p=n s$ ) and BMI (group $A=29 \pm 5 \mathrm{~kg} / \mathrm{m} 2$ versus group $B=28 \pm 5 \mathrm{~kg} / \mathrm{m} 2, \mathrm{p}=\mathrm{ns}$ ).

Conclusions. Our study confirms previous observations. Nevertheless it needs to consider that group A, in our study, is also characterized by a greater LVMI; then it is possible to deduce that the ventricular repolarization can be conditioned by LVMI more than left ventricular asymmetry. 